\title{
Servo signal processing for flying height control in hard disk drives
}

\author{
Uwe Boettcher • Christopher A. Lacey • \\ Hui Li $\cdot$ Kensuke Amemiya . \\ Raymond A. de Callafon - Frank E. Talke
}

Received: 31 August 2010/Accepted: 16 December 2010/Published online: 7 January 2011

(C) The Author(s) 2011. This article is published with open access at Springerlink.com

\begin{abstract}
A novel method of measuring the relative headmedium spacing based on a measurement in the servo sectors is developed and simulated using a read back signal model. The spacing measurement is tested experimentally on a spin stand where the flying height is varied using the resistance heater element in a thermal flying height control slider. In addition, voltage step response measurements were obtained. The data were used to perform system identification and estimate the dynamics of the thermal actuator. The model can potentially be used for thermal flying height control.
\end{abstract}

\author{
U. Boettcher $(\bowtie) \cdot$ R. A. de Callafon · F. E. Talke \\ Center for Magnetic Recording Research, \\ University of California, San Diego, \\ 9500 Gilman Drive \# 0401, La Jolla, CA 92093, USA \\ e-mail: uwe@ucsd.edu \\ R. A. de Callafon \\ e-mail: callafon@ucsd.edu \\ F. E. Talke \\ e-mail: ftalke@ucsd.edu \\ C. A. Lacey \\ Microphysics, Inc., 4620 Fortran Dr., Suite 120, \\ San Jose, CA 95134, USA \\ e-mail: chrislacey@microphysics.com \\ H. Li - K. Amemiya \\ Storage Mechanics Laboratory, Hitachi Asia Ltd., \\ Singapore 049318, Singapore \\ e-mail: hli@has.hitachi.com.sg \\ K. Amemiya \\ e-mail: kamemiya@has.hitachi.com.sg
}

\section{Introduction}

In recent years, thermal actuated flying height control has been implemented in hard disk drives (HDD) to reduce the effect of flying height variations due to manufacturing tolerances or write current induced pole tip protrusion (Gong and Liu 2005). A stable low flying height is necessary to achieve low bit error rates (Tang et al. 2007). Thermal flying height control is accomplished through a resistance heater element in close proximity to the read write element. Applying a voltage to the heater element causes a thermally induced deformation of the air bearing surface which causes a reduction in magnetic spacing and flying height at the read write element (Fig.1). Thermal flying height control is used in a 'static manner' in today's disk drives, i.e., a constant power is applied to the heater during writing and reading, respectively. Dynamic flying height variations of a slider over a disk are composed of repeatable and non-repeatable contributions. As the name suggests, repeatable variations of flying height occur at the same angular and radial position of the slider above the disk at each revolution (Xu et al. 2006). Thus, the question arises as to whether a thermal flying height control slider can be used to dynamically control the repeatable flying height variations between slider and disk. One of the first approaches to dynamic flying height has been shown in (Shiramatsu et al. 2008) where time domain and frequency domain data have been used to estimate the flying height signal.

It is also likely that, as the flying height is decreased, contact between slider and disk occurs at the same position on the disk. Therefore, thermal flying height control could be used to dynamically increase the flying height in those regions as the overall flying height could be decreased to the lowest stable flying height. The objectives of this paper 


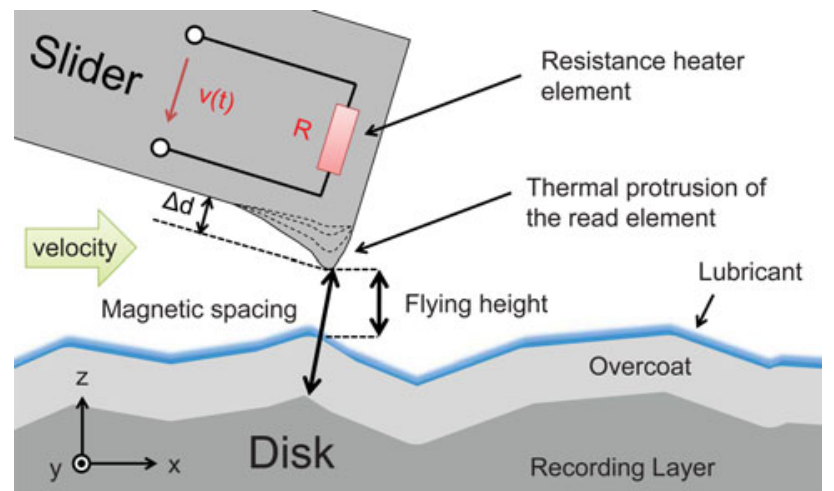

Fig. 1 Side view of the trailing edge of the slider and the top layers of the disk

are to propose a method for accurately measuring the flying height variations based on servo sector measurements.

\section{Flying height estimation}

\subsection{Available techniques}

Most of the reported algorithms on in-situ flying height estimation are based on a specific data pattern that is written onto the disk; thereby resulting in certain harmonics in the frequency spectrum of the read signal. The Wallace spacing formula translates the decay of the signal to spacing change and has been shown to be applicable to longitudinal magnetic recording as well as perpendicular magnetic recording (Guarisco et al. 2006). Commonly used is the triple harmonics method ( $\mathrm{Xu}$ et al. 2006; Liu and Yuan 2000; Yuan et al. 2002) that uses a "111100" data pattern to create a large first and third harmonic. The logarithm of the ratio of the third over the first harmonic is proportional to the flying height modulation. Ratios of different harmonics other than the third and the first have also been used and servo pattern might be taken into account (US Patent Application 20090153996). Approaches have also been implemented based on maximum or average amplitudes (US Patent 7522360) rather than harmonics amplitudes. Also, approaches that employ random data instead of a fixed data pattern were proposed (Zeng and Chen 2003). A technique that extracts both the position error signal (PES) and the flying height information is reported in (US Patent 7253984) where radially adjacent and circumferentially aligned servo bursts are generated with different frequency contributions to generate position error and flying height signals. Other techniques are the pulse width method (Klaassen and van Peppen 1994), the spectral fitting method (Fu et al. 2007) and methods that estimate the flying height based on the slope of isolated pulses of the read back signal (US Patent 6288856). Some

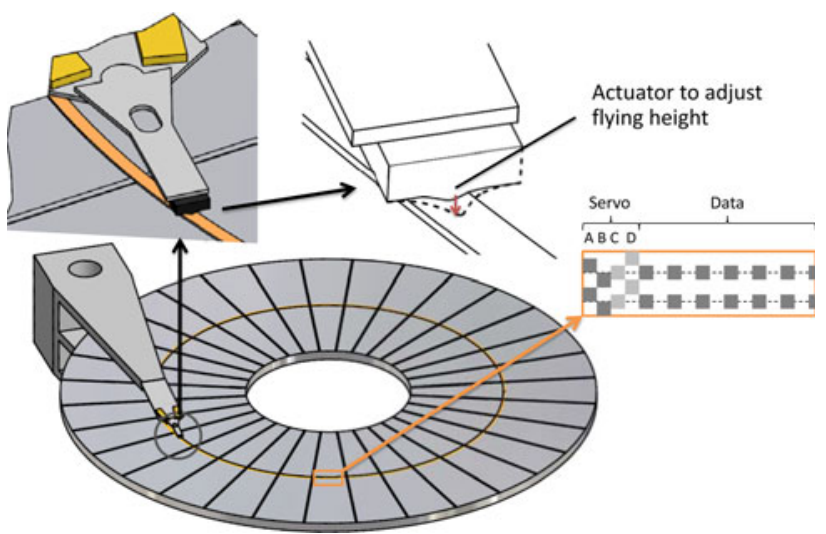

Fig. 2 Disk with servo and data sectors

of these estimation algorithms are only applicable to longitudinal magnetic recording (LMR) technology and some are developed for (or extendable to) perpendicular magnetic recording (PMR) technology. Some embody the following disadvantages:

- Cross-track-motion might wrongfully be detected as a change in flying height

- The measurement can strongly depend on the radial position of the read element over the disk (skew angle)

- A particular data pattern and/or the data sector is necessary, and, thus, storage space is lost.

- The method may not be capable for perpendicular magnetic recording

\subsection{Servo signal based flying height estimation}

State-of-the-art HDDs have typically an embedded servo as indicated in Fig. 2. The servo sectors on the disk are used to determine the off-track position of the read/write head with respect to the track center. The estimation scheme for the measurement of the variation of flying height proposed in this paper is based on the servo pattern written in the servo sectors on the disk. Using the servo pattern for the estimation of flying height has a number of advantages. At every servo sector the off-track position is known. The PES is generated using a pre-written servo pattern on the disk. Hence, the effect of cross-track motion of the head on the flying height signal can be eliminated. The servo can be an amplitude based servo or a timing based servo. Timing based servo applications have been shown to be more accurate than amplitude based counterparts (Han and de Callafon 2009). The estimation of flying height variation using a conventional amplitude based servo pattern will be shown in this paper. The following assumptions are made:

1. The change in magnetic spacing corresponds to a change in flying height which requires a constant overcoat and lubricant thickness (Fig. 1) 
2. The flying height variation of adjacent servo bursts within the same servo sector is small compared to the flying height variation between adjacent servo sectors.

3. The writing process is less sensitive to flying height variations than the reading process (Yuan et al. 2002)

Based on those assumptions the change in flying height can be computed from the Wallace equation. The Fourier transform of the read back signal decays exponentially with increasing distance from the magnetic medium (Bertram 1994). For simplicity, only A and B bursts are considered here. The read back signal voltage is measured at a specific frequency in the frequency domain of two subsequent servo bursts, i.e. $\Phi_{A}$ and $\Phi_{B}$

$\Phi_{A}(k, z)+\Phi_{B}(k, z)=\left(\Phi_{A}(k, 0)+\Phi_{B}(k, 0)\right) e^{-k z}$

where $k=\frac{2 \pi}{\lambda}$ is the wave number. The flying height change $\Delta z=z-z_{\text {ref }}$ can be calculated from

$\Delta z=-\frac{\lambda}{2 \pi} \ln \left(\frac{\Phi_{A}(\lambda, z)+\Phi_{B}(\lambda, z)}{\Phi_{A}\left(\lambda, z_{\text {ref }}\right)+\Phi_{B}\left(\lambda, z_{\text {ref }}\right)}\right)$.

The fluctuation of the product of the recording layer thickness and the remanent magnetization $\left(M_{\mathrm{rt}}\right)$ of the recording media (Yuan et al. 2002) causes read back signal modulation, and, therefore modulations in the measured flying height. The harmonics ratio method referred to earlier decreases this effect. Two different approaches are proposed here, both based on the first and the third harmonic of the read back signal in the servo sector. We can modify Eq. 1 to compute the first harmonic corresponding to the wavelength $\lambda_{1}$

$\ln \left(\Phi_{A 1}+\Phi_{B 1}\right)=\ln \left(\Phi_{0, A 1}+\Phi_{0, B 1}\right)-\frac{2 \pi}{\lambda_{1}} z$

and the third harmonic corresponding to the wavelength

$\ln \left(\Phi_{A 3}+\Phi_{B 3}\right)=\ln \left(\Phi_{0, A 3}+\Phi_{0, B 3}\right)-\frac{6 \pi}{\lambda_{1}} z$

Now, Eq. 4 can be subtracted from Eq. 3 and the change in flying height can be computed via
$\Delta z_{1-3}=+\frac{\lambda_{1}}{4 \pi}\left(\ln \left(\Phi_{A 1}+\Phi_{B 1}\right)-\ln \left(\Phi_{A 3}+\Phi_{B 3}\right)\right)$

which is similar to the known triple harmonics formula except that the sum of servo burst harmonics are considered. The subscript " $1-3$ " indicates that the difference between the first and third harmonic was taken into account for the computation of $\Delta_{z}$. Alternatively, the sum of Eqs. 4 and 3 can be used to calculate

$\Delta z_{1+3}=-\frac{\lambda_{1}}{8 \pi}\left(\ln \left(\Phi_{A 1}+\Phi_{B 1}\right)+\ln \left(\Phi_{A 3}+\Phi_{B 3}\right)\right)$

\subsection{Simulated off-track characteristics}

The cross-track characteristics of the flying height change estimate were simulated using the same model and parameters as in (Boettcher et al., unpublished data) for three cases $(18,10$ and $2 \mathrm{~nm})$ for LMR and PMR, respectively. Two different servo burst spacing values were simulated. A servo burst spacing of 0.5 (Fig. 3a, b) corresponds to a shift of $0.5 \mathrm{~W}$ from the track center for each burst. The resulting total radial distance between $\mathrm{A}$ and $\mathrm{B}$ burst would yield $0.5 W+0.5 W=1 W$ which equals the bit width. A servo burst spacing of 0.25 (Fig. 3c) indicates a $50 \%$ overlap of the servo bursts.

The flying height change in Fig. 3 was estimated based on Eqs. 5 and 6 as given by the dash-dot line and the dashed line, respectively. Since the measurement is relative, it was shifted in the $\mathrm{z}$ direction to the $2 \mathrm{~nm}$ track center value. The magnetic spacings at 10 and $18 \mathrm{~nm}$ were computed using the relative knowledge of the $2 \mathrm{~nm}$ value. It can be seen that the 0.5 burst spacing case has a very small sensitivity to off-track variations for both LMR and PMR (Fig. 3a, b). The two proposed methods measure almost the same value close to the track center. However, the values of $\Delta z_{1-3}$ and $\Delta z_{1+3}$ differ slightly but the arithmetic mean (solid line) of both yields the correct values (10 and $18 \mathrm{~nm}$ ) close to the track center. This effect becomes more obvious for a burst spacing of 0.25 as shown
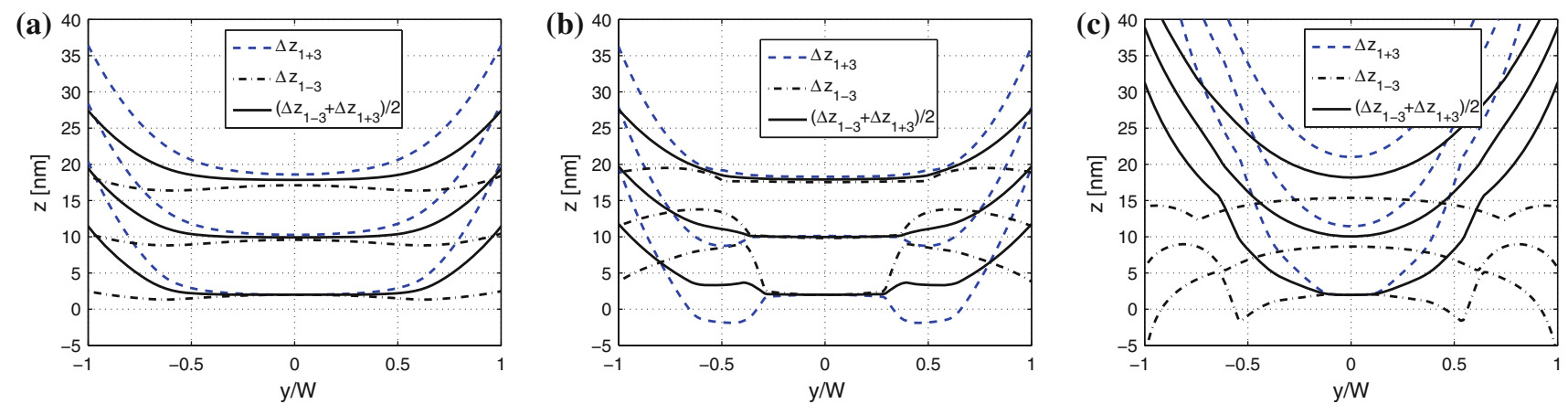

Fig. 3 Computed head-medium-spacing $z$ for 18,10 and $2 \mathrm{~nm}$ and burst spacing of a 0.5 and longitudinal recording b 0.5 and perpendicular recording $\mathbf{c} 0.25$ and perpendicular recording as a function of the normalized off-track position $y$ 
in Fig. 3c. The arithmetic mean of Eqs. 6 and 5 simply yields

$\Delta z_{\text {mean }}=\frac{\lambda_{1}}{16 \pi}\left(\ln \left(\Phi_{A 1}+\Phi_{B 1}\right)-3 \ln \left(\Phi_{A 3}+\Phi_{B 3}\right)\right)$

Based on the performed simulations, the relative flying height change computed by Eq. 7 is relatively insensitive to off-track motion for $|\Delta x|<0.3 \mathrm{~W}$ and a burst spacing of 0.5 . For a burst spacing of 0.25 it is still relatively insensitive to off-tack motion for $|\Delta x|<0.1 W$ which is the generally accepted positioning requirement in magnetic recording technology in order to avoid track misregistration. A sudden increase in difference between $\Delta z_{1-3}$ and $\Delta z_{1+3}$ might indicate large off-track motion. However, $\Delta z_{\text {mean }}$ shows small off-track sensitivity compared to $\Delta z_{1-3}$ and $\Delta z_{1+3}$ within a much larger range.

\section{Experimental results}

\subsection{Experimental set-up and methodology}

A number of flying height modulation measurements have been performed using a disk head tester (MicroPhysics). The experimental set-up is shown in Fig. 4. Perpendicular magnetic recording disks and matching head-gimbal-assemblies (HGA) were employed. The disk was spun at 7,200 rpm and a simple dual servo pattern with $\mathrm{A}$ and $\mathrm{B}$ bursts only was written onto the disk using a 16T pattern. A write frequency of $900 \mathrm{MHz}$ was used with the first harmonic of the servo occurring at $56.25 \mathrm{MHz}$. The read back signal was captured using a $2 \mathrm{GS} / \mathrm{s}$ digitizer. All measurements were performed at $2^{\circ}$ skew angle and at a radius $r=25 \mathrm{~mm}$. The disk was divided into 128 servo sectors which yields a sampling frequency of position error signal and flying height measurement of $15.36 \mathrm{kHz}$. As indicated in Fig. 4, an arbitrary waveform generator was used to apply a voltage to the thermal actuator and a spindle index signal was used as a trigger for the waveform generator.

\subsection{Verification of proposed flying height estimation scheme}

The proposed estimation method from Section 2 was used to calculate the flying height modulation based on the measured read back signal. First, the dynamic flying height modulation was measured for constant (but gradually increased) power levels. Figure 5 shows 15 different heater power levels $P_{\text {in }}$ and the flying height modulations were computed based on Eqs. 5 and 6, respectively.

The PES was computed by

$\mathrm{PES}=\frac{A-B}{A+B}$

where $A$ and $B$ represents the track averaged amplitude (TAA) of the A and B burst, respectively. In Fig. 5, averaged values of 20 measurements are shown. It should be noted that a voltage $v$ was applied to the heater element and the resulting input power $P_{\text {in }}$ was computed by $P_{i n}=\frac{v^{2}}{R}$ assuming a fixed value of the heater resistance $R$. Subsequently, we investigate the effect of resistance change in the DC gain of the model. $P_{\text {in }}$ was varied from $0 \mathrm{~mW}$ to approximately $130 \mathrm{~mW}$. From Fig. 5 it can be observed that the measured relative flying height decreases as the heater power increases. It is furthermore observed that the dynamic variation of $\Delta z$ is very repeatable and very similar for different bias power level except for a DC offset. The difference between the static flying height variation $\Delta z_{1+3}$ and $\Delta z_{1-3}$ is very small. However, in terms of dynamic behavior, $\Delta z_{1+3}$ looks slightly different from $\Delta z_{1-3}$ at some angular positions. In general, $\Delta z_{1+3}$ shows more low frequency flying height variations compared to $\Delta z_{1-3}$.

Fig. 4 Experimental set-up

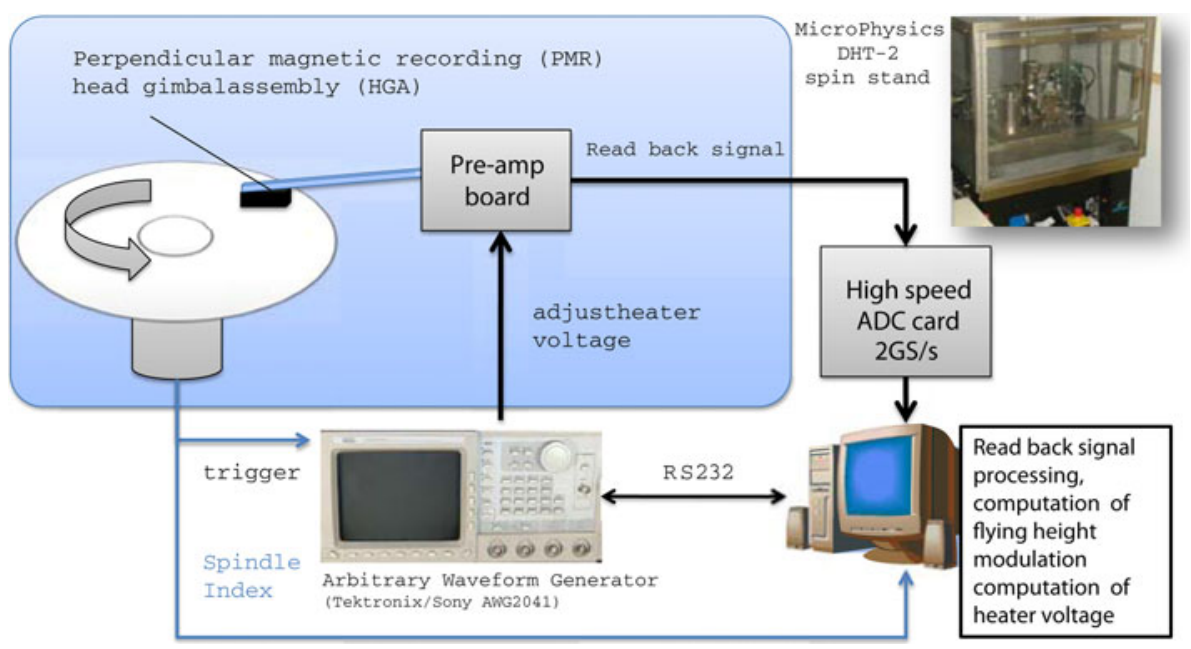


Fig. 5 Averaged flying height modulation for 15 different constant power inputs to the heater element and position error signal over one revolution
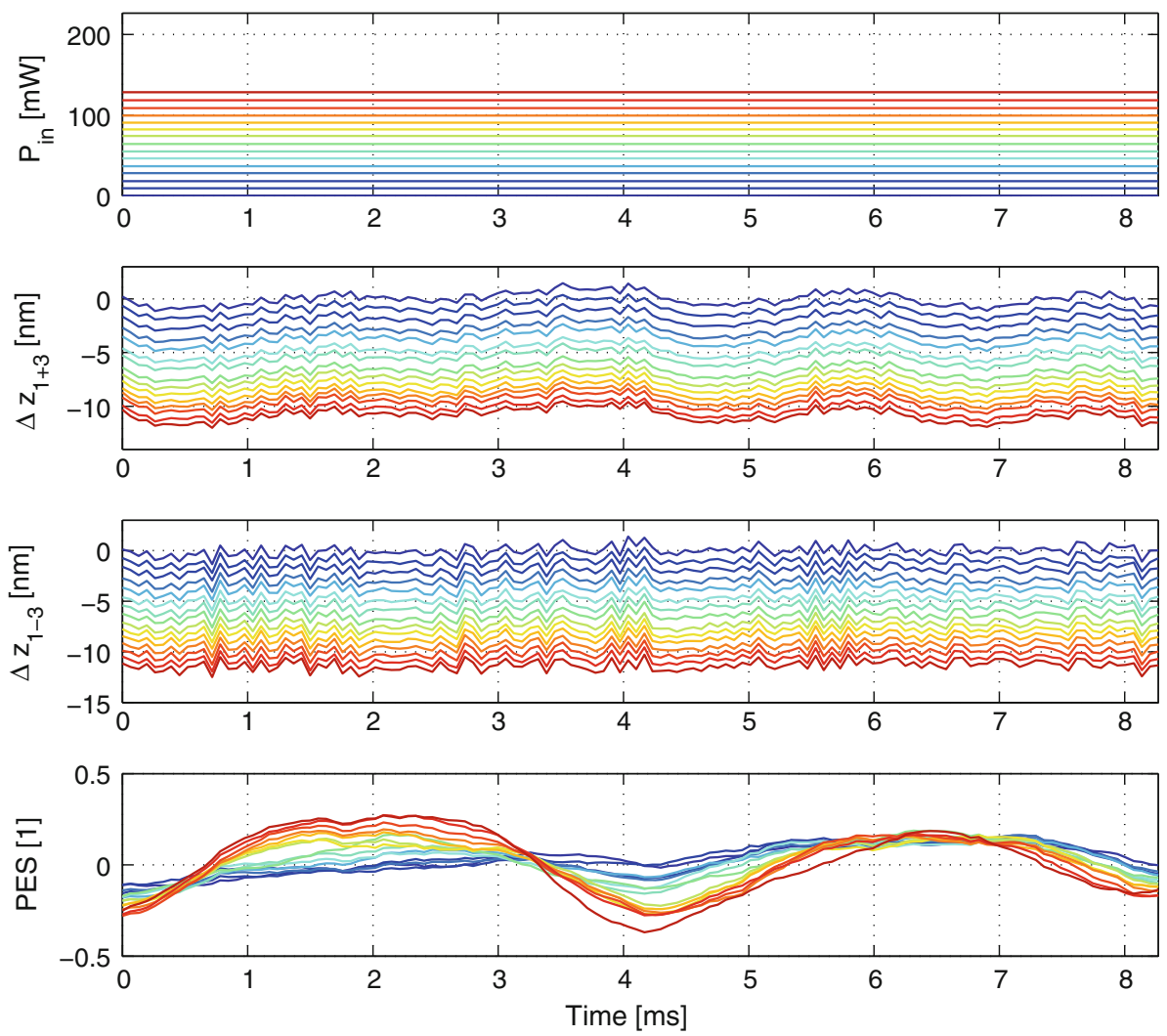

(a)

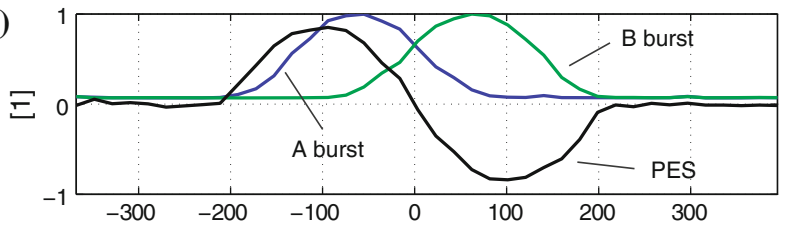

(b)

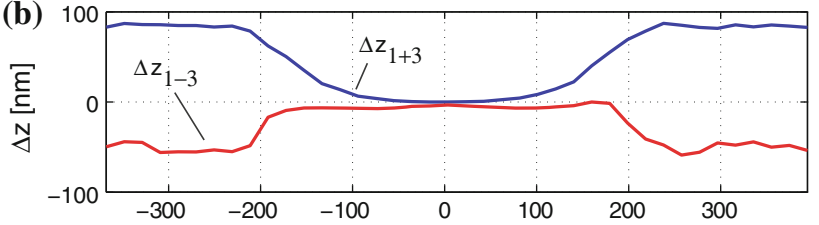

(c)

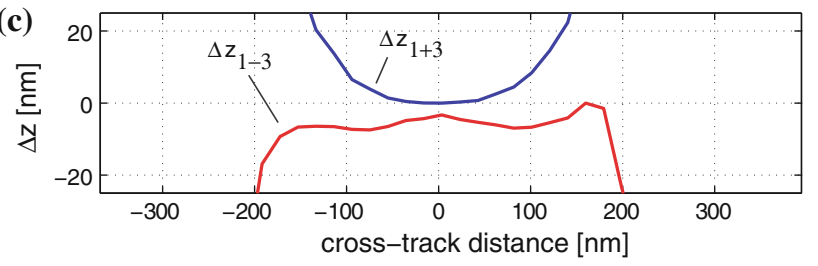

Fig. 6 Measured cross-track behavior at $10 \mathrm{~mW}$ heater input. a track averaged amplitude (TAA) for A burst an B burst, $\mathbf{b}$ measured flying height modulation, $\mathbf{c}$ zoomed version of $\mathbf{b}$

\subsection{Off-track characteristics}

A single (non-averaged) off-track measurement is shown in Fig. 6. Here, Fig. 6a shows the track averaged amplitude of the $\mathrm{A}$ and $\mathrm{B}$ burst and the computed PES according to Eq. 8. On a large scale (Fig. 6b) $\Delta z_{1-3}$ appears less sensitive to off-track motion than $\Delta z_{1+3}$. However, on a smaller scale (Fig. 6c) it can be observed that $\Delta z_{1+3}$ is quite insensitive to off-track motion close to the track center. As predicted by the simulation results in Sect. 2, an increase in measured flying height can be observed for increasing off-track motion for $\Delta z_{1+3}$ where $\Delta z_{1-3}$ decreases for increasing off-track motion.

\subsection{Voltage step measurements}

In order to identify a dynamic model of the heater actuator, a step input was applied. The results are shown in Fig. 7. The step height of the input voltage was kept constant and only the bias voltage was increased yielding a linearly increasing power step height with increasing bias voltage. As in the static case, an average over 20 measurements was calculated. Figure 7 shows that the response of the actuator is very fast and that there is a minimum flying height around $12 \mathrm{~nm}$ below the initial flying height. The flying height does not go below this limit which is likely the result of head/disk contact. For heater power values above approximately $80 \mathrm{~mW}$ a sudden increase in position error signal and variance in flying height modulation (Fig. 8) can be observed which might indicate head disk contact. 
Fig. 7 Averaged flying height modulation for 15 different step power inputs to the heater element and position error signal over one revolution
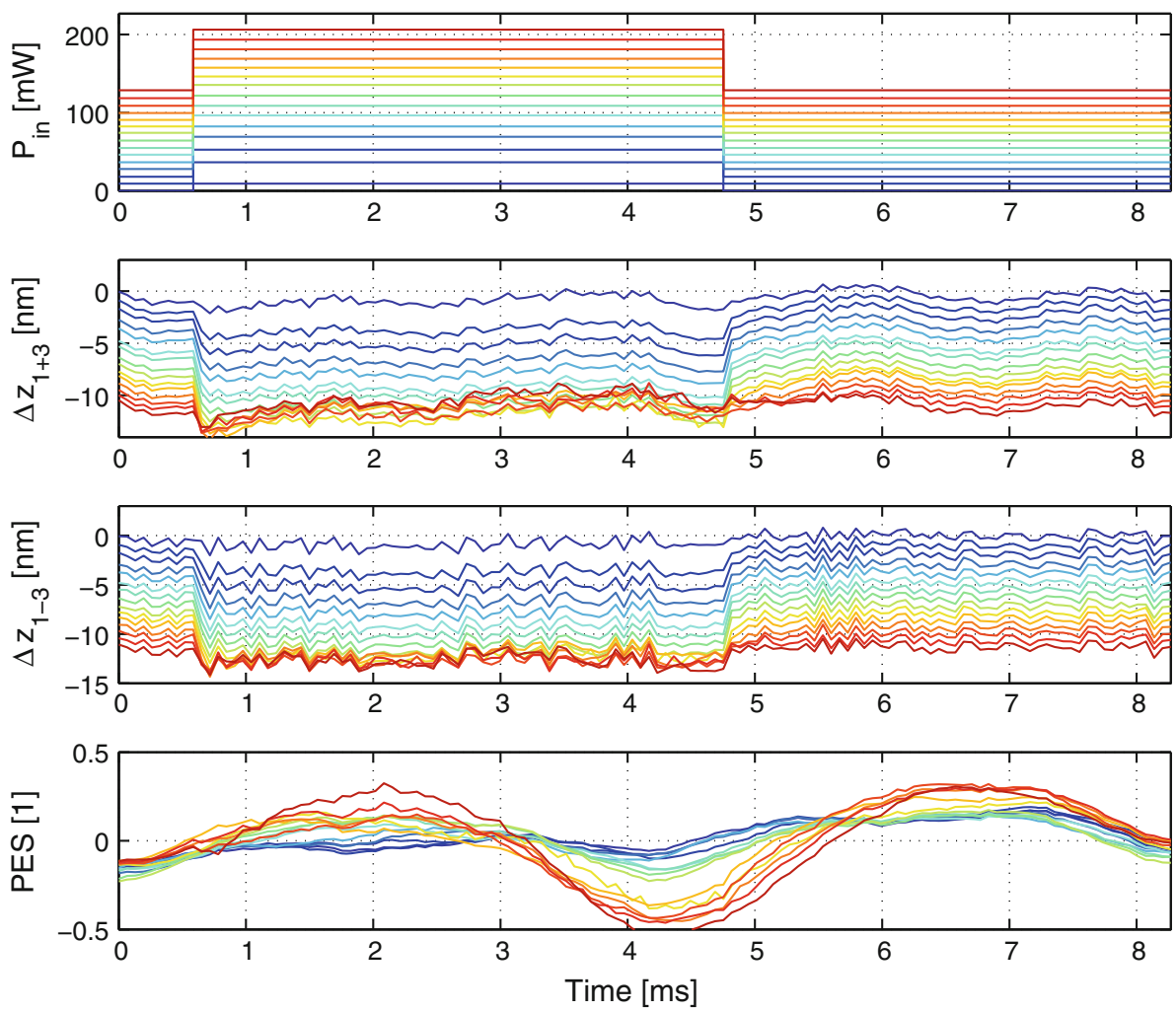
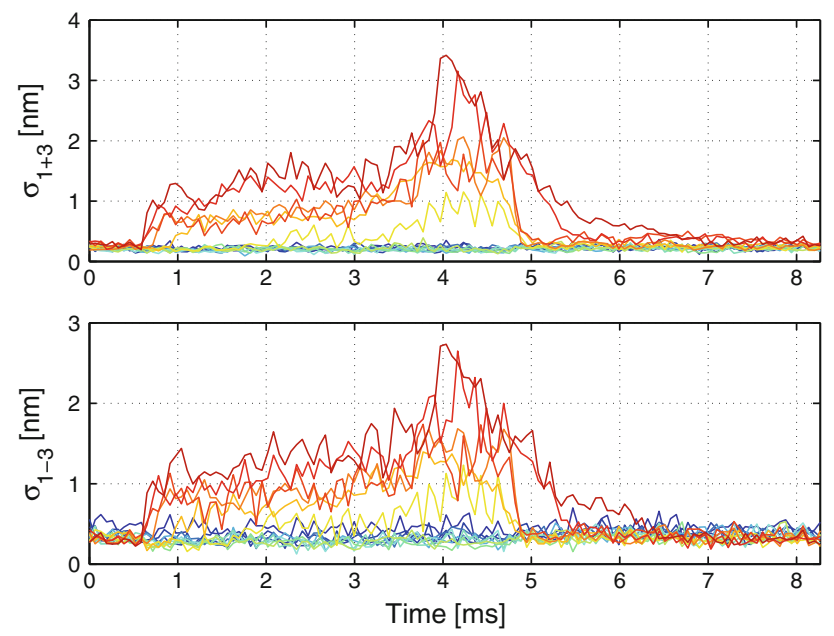

Fig. 8 Variance in relative flying height

\subsection{Data based dynamic modeling of the heater response}

The heater response was identified based on the relative spacing between the reference measurement (Fig. 5) and the step measurement (Fig. 7). The relative spacing measurements for the 15 different input steps are shown in Fig. 9. The two different estimation methods are in excellent agreement. The heater response was modeled using a standard least-squares estimation and a first order model. A significant time-delay could not be measured since the sampling time the of flying height modulation measurement was $65.1 \mu \mathrm{s}$ which is on the order of the determined time constant $\tau$. It is obvious that this results in a decreased accuracy of the estimation of $\tau$ compared to the estimation accuracy of the steady-state gain $K$ based on the least-squares solution. Time constant $\tau$ and gain $K$ were estimated for both proposed relative flying height estimation methods and Fig. 10 shows the results as a function of the power step size $P_{\text {step }}$. The average time constant for the measurements up to $80 \mathrm{~mW}$ bias power $P_{i n}$ is on the order of the PES sampling time (65 $\mu \mathrm{s})$. The absolute value of the gain $K$ decreases from about $0.1 \mathrm{~nm} / \mathrm{mW}$ gradually to almost zero as the bias heater power $P_{i n}$ and step size $P_{\text {step }}$ increases. This is likely due to contact with the disk at approximately $12 \mathrm{~nm}$ where a further head-disk-distance decrease is not possible. The static flying height change as a function of heater input power $P_{i n}$ is shown in Fig. 11. The relationship seems nearly linear, although the slope of the curve (which corresponds to the static gain) is slowly decreasing as $P_{\text {in }}$ increases. This could be due to the air bearing cooling effect (increased thermal conductivity between head and disk as local air bearing pressure increases and flying height decreases) (Chen and Bogy 
Fig. 9 Relative spacing measurements (Fig. 5 subtracted from Fig. 7) for 15 different input step sizes (crosses) and identified first order models (solid lines)
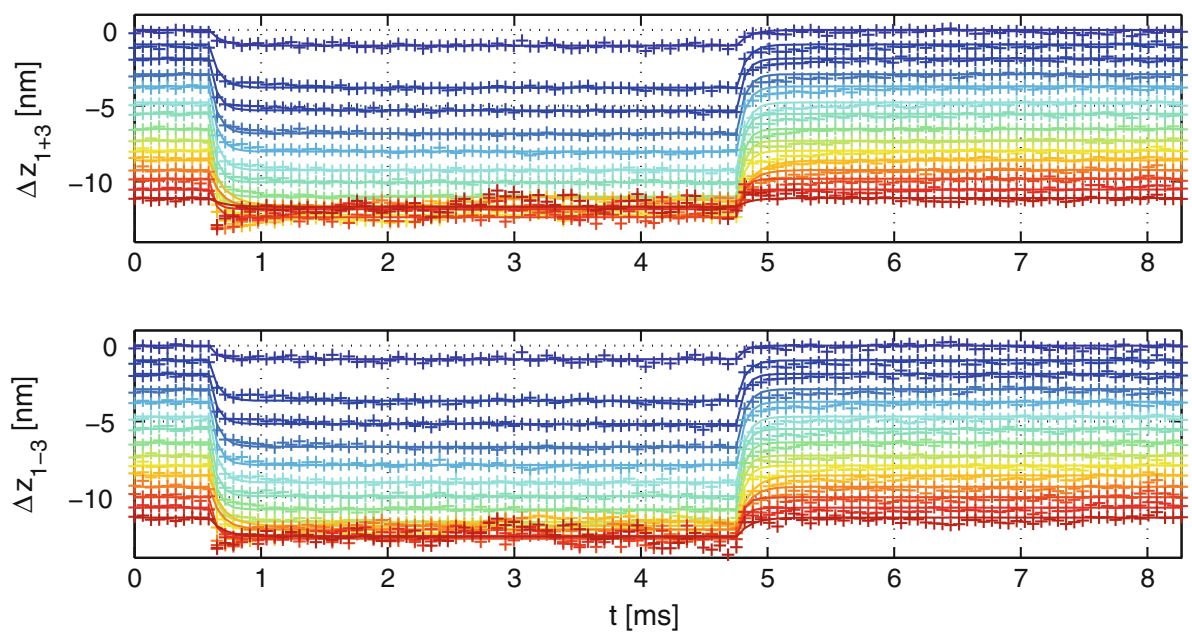
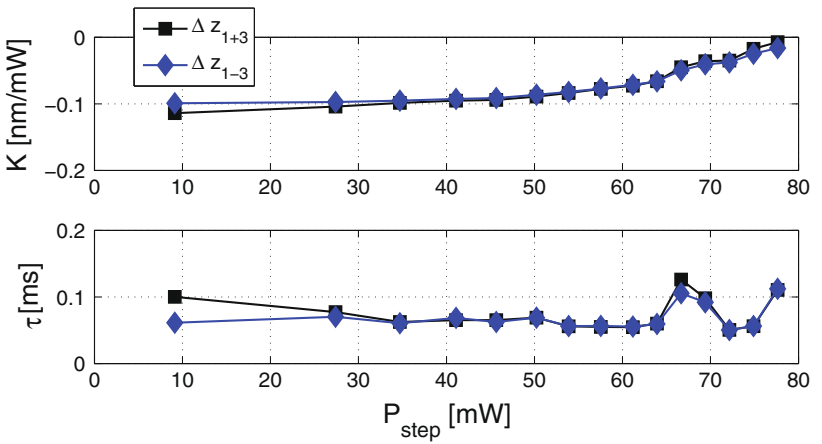

Fig. 10 Estimated gain $K$ and time constant $\tau$ from Fig. 9 based on a least squares estimate

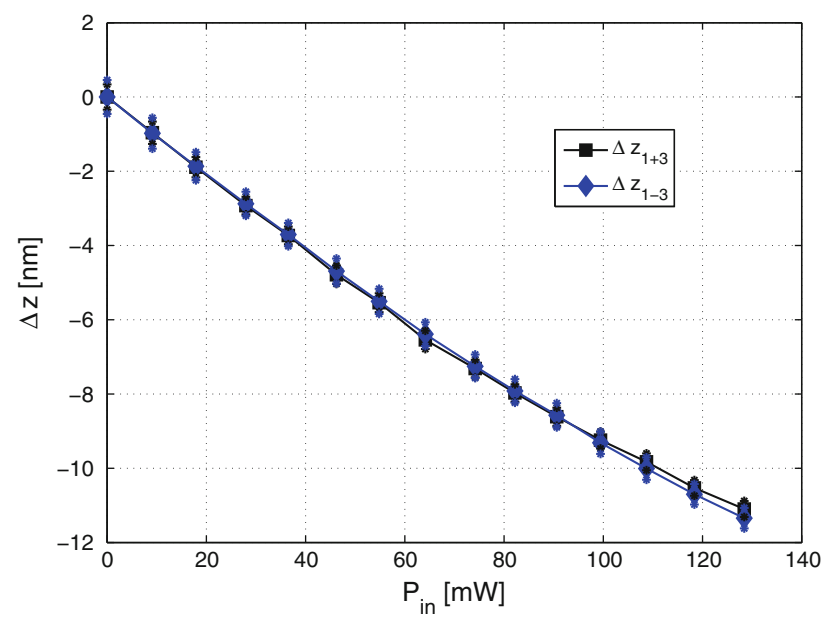

Fig. 11 Static flying height change for different heater input power levels

2008) and/or the so-called push-back effect (due to higher air bearing pressure at lower flying heights) (Zhou et al. 2010). Another explanation would be an increasing heater resistance with increasing heater voltage yielding an actual lower heater power compared to the computed heater power assuming a constant resistance.

\section{Conclusions}

A flying height measurement scheme based on the servo pattern recorded in the servo sectors was developed and simulated using a read back signal model. The two proposed flying height measurement methods show excellent agreement within a large flying height range while being insensitive to off-track motion of the read write head. In the proposed method, the off-track sensitivity is a function of the radial servo burst spacing, i.e., the cross-track spacing between the A and B burst in each servo sector. The proposed method was tested experimentally to identify the dynamics of a resistance heater element capable of modifying the flying height at the read/write element. Based on the current data that uses a servo sector-based signal at a sampling rate of approximately $15.36 \mathrm{kHz}$ it can be concluded that the response of the thermal actuator can be approximated by a first order system with a time-delay. It was found that the time delay was negligible and the time constant was found to be very small at around $65 \mu$ s. This promises feasible solutions to real-time flying height adjustment up the 15-20 kHz-regime. The gain or actuator efficiency (input power vs. flying height reduction) decreases as the flying height decreases which might be due to the push-back effect or the air-bearing cooling effect.

Acknowledgments We would like to thank Gregory Kimball of Texas Instruments, John Contreras of Hitachi Global Storage Technologies, Alexei Sacks of Seagate Technology and Tom Crittenden of Microphysics for their interest in this work and for their help with the experimental set-up.

Open Access This article is distributed under the terms of the Creative Commons Attribution Noncommercial License which 
permits any noncommercial use, distribution, and reproduction in any medium, provided the original author(s) and source are credited.

\section{References}

Bertram N (1994) Theory of magnetic recording. University Press, Cambridge, $\mathrm{p} 40$

Chen D, Bogy D (2009) Simulation of static flying attitudes with different heat transfer models for a flying-height control slider with thermal protrusion. Tribol Lett. Springer, Netherlands, pp 1-9

Fu C, Takeo A, Bertram HN (2007) Technique to obtain headmedium magnetic spacing in a disk drive. J Appl Phys 10P107

Gong ZQ, Liu JJ (2005) Pole-tip protrusion effect on head-disk interface at low flying clearance. IEEE Trans on Magn 41(10):3019-3021

Guarisco D, Li Z, Higgins BE, Saito K, Wu Y, LeFebvre A (2006) Drive integration in perpendicular recording (invited). J Appl Phys 99:08Q908

Han Y, de Callafon RA (2009) Evaluating track-following servo performance of high-density hard disk drives using patterned media. IEEE Trans Magn 45(12):5352-5359
Klaassen KB, van Peppen JCL (1994) Slider-disk clear-ance measurements in magnetic disk drives using the readback transducer. IEEE Trans Instr Meas 43(2):121-126

Liu B, Yuan Z (2000) Tribo-magnetics and nanometer spaced headdisk systems. Asia-Pacific Magnetic Recording Conference TB5/ $1-\mathrm{TB} 5 / 2$

Shiramatsu T, Atsumi T, Kurita M, Shimizu Y, Tanaka H (2008) Dynamically controlled thermal flying-height control slider. IEEE Trans on Magn 44(11):3695-3697

Tang Y, Hong SY, Kim NY, Che X (2007) Overview of fly height control applications in perpendicular magnetic recording. IEEE Trans on Magn 43(2):709-714

Xu J, Shimizu Y, Su L (2006) Drive level measurement of flying height modulation and control of slider disk contact. Tribol Lett 24(2):159-162

Yuan ZM, Liu B, Zhang W, Hu SB (2002) Engineering study of triple-harmonic method for in situ characteriza-tion of head-disk spacing. J Magn Magn Mat 239(1-3):367-370

Zeng QH, Chen M (2003) Flying height modulation estimation from pseudorandom readback signal in disk drives. IEEE Trans on Magn 39(5):2417-2419

Zhou W, Liu B, Yu S, Hua W, Wong C (2010) Effects of environmental temperature and humidity on thermal flying height adjustment. Microsyst Technol 16(1):49-55 